\title{
AC and DC caracteristics of simulated Doped Graphene Field Effect Transistor (GFET) Frequency Multipliyer
}

\author{
Rohit Nimje ${ }^{1}$, Rabinder Henry ${ }^{2}$, Amit Patwardhan ${ }^{2}$, Jayant Pawar ${ }^{2}$, \\ Prakash Viswanathan ${ }^{2}$, Ashish Kumar Patel ${ }^{2}$, Pratik More ${ }^{2}$ \\ ${ }^{1}$ Center for Nanotechnology Research, VIT University, Vellore, Tamil Nadu, India. \\ ${ }^{2}$ Hope Foundation's, Pralhad P. Chhabria Research Center (PPCRC), Pune, Maharashtra, India.
}

\begin{abstract}
The presented work demonstrates the simulation of Graphene Transistor. Graphene filed effect transistor can be operated at high-frequency. The material parameters for device simulation have been extracted from the recently published literature. The device carrier transport calculations are performed with semiclassical Boltzmann transport models along with Poisson equations. The current-voltage characteristics analyzed and performance of the device is evaluated. Device parameters of the graphene radio frequency transistor have been extracted. The equivalent circuit has been built with passive components by using general purpose PSpice circuit simulator. Then the equivalent circuit performance is analyzed for a given input frequency.
\end{abstract}

Keywords: GFET, Semiconductor devices, modeling, Simulation, etc.

\section{INTRODUCTION}

Doped Graphene Filed Effect Transistor (GFET) improves career transport phenomenon [23].This is prorperty enables to utilize such marterials for development of high-performance application devices such as RF mobile unit, satellite communication and military applications [11, 14, 34]. GFET amplifier perform well on encoding and decoding for a high frequency signal $[32,45]$. Graphene FET have four contacts such as drain, source, top gate and bottom substrate and each terminal has specific operational functionality. Works [36, 41].

GFET operaions are similar to FET (Field Effect Transistors).Gate controls the electron flow through channel with respect to bottom substrate $[10,30]$.This dertermines the nature of oerpation in the GFET reuslting in enhancement mode. GFET frequency amplifiers transport high electron density at low power which is major advantgae compared to to High Electron Mobility Transistor (HEMT) [1, 12].

Graphene is a single crystalline two-dimensional material formed by mechanical exfoliation methods from graphite. It occupies $\mathrm{sp}_{2}$ hybridization of $0.34 \mathrm{~nm}$ thick carbon atoms [42]. Lattice structure is formed by these carbon atoms [22]. The pure condcuting nautre of the Grpahene is doped to develop new type of electronic devices [35]. Since oped graphene behaves like a semicondcutor [1].

There is need to occupy narrow bandgap in the graphene sheet. doped graphene bandgap is engineered by improving defect in the graphene. Graphene occupies narrow bandgap between conduction and valance band. The ambipolar behavior of GFET operates on high electron mobility transport $[2,20]$.
The device simualtions are perfomed using ATLAS deisgn tool.Well-designed GFET on TCAD Silvaco by extracting material parameters [1-3, 48]. GFET mirrior with MOSFET are replaced in silicon substrate with hexagonal silicon carbide material $[13,17,33]$. This results in quantum confinement channel by high dielectric material like hafnium dioxide $\left(\mathrm{HfO}_{2}\right)$ as gate capacitance [5]. Simulation of GFET with shorter channel length improves large career transport current thorugh doped graphene channel. Larger the current, Less requirement of gate voltage, high transconductance and high output voltage improves performance of GFET is depends on their DC analysis [16]. GFET device parameters are well extracted in small signal equivalent circuit on circuit simulator tool. A lower intrensic gate capacitance are imforms to take place GFET into the high frequency application [25]. This GFET device were tested in $10 \mathrm{MHz}$ input frequency, its ransmitted at a maximum of $26 \mathrm{MHz}$. GFET improves performace of frequency multiplier output. Later on it will used in band pass filter and frequency seperation units [19].

\section{DESIGN METHODOLOGY}

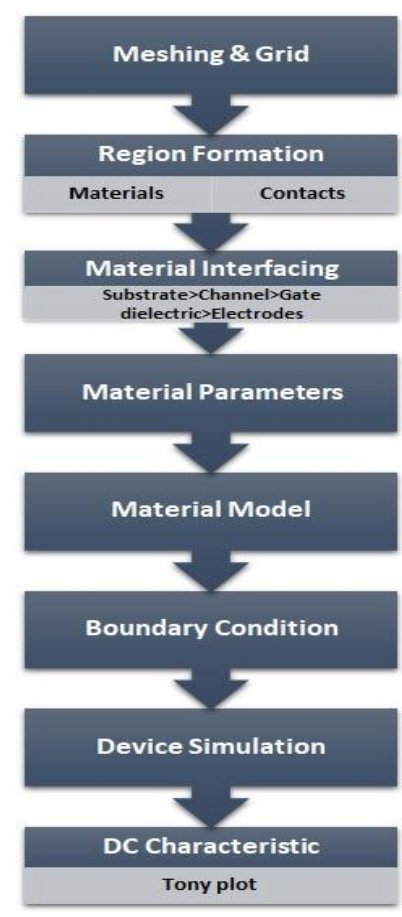

Figure 1: Computational modeling steps for GFET modeling

GFET device is structured and defined with Atlsa device simulator. Computational work starts from meshing, griding, 
region formation, interfacing, Contacts identifications, material interface, material parameters, material model, boundary condition, Numerical method, Device simulation, DC caracteristics and ploting, etc. as shown in Figure 1 Silvaco/Atlas TCAD tool provies user friendly computional facility $[15,48]$. The material constants like doped graphene bandgap, , dielectric values has been extracted from published research papers [27]. The GFET device is modeled by extracting device parameteres such as material constant, gate length, length of oxide thickness and channel width, etc. [4]. in nanometer scale. This is applicable for high career mobility transport also and depeneds on shorter channel length. [22, 37, 41].

\section{MATERIALS AND METHODS}

The selection of material reflects the device performance and includes doped graphene parameters such as energy band gap, permittivity, affinity, a material mobility of doped graphene extracted from research articles mention Table 1. There are various types of graphene bandgap opening such as substrate induced band gap opening, chemical substitution doping, hybrids, quantum confinement nanoribbon GNR (zig-zag and armchair), Graphene Nano-mesh, etc. [8]. $0.25 \mathrm{eV}$ energy bandgap is utilized from recently published research papers [12]. GFET device modeled by metal oxide semiconductor FET. For the exploration of high electron and hole mobility through at $200 \mathrm{~nm}$ GNT channel length depends on quantum confinement of the device. It requires high dielectric gate material hafnium dioxide $\left(\mathrm{HfO}_{2}\right)$.

Table 1: Doped graphene channel material parameter

\begin{tabular}{|c|c|}
\hline Parameter & Doped graphene \\
\hline Energy Band Gap & $0.25 \mathrm{eV}$ \\
\hline Concentration (nc300/nv300) & $2.5 \times \mathrm{x} \mathrm{e}^{20}$ \\
\hline Mobility & $12000 \mathrm{~cm} /(\mathrm{V}-\mathrm{s})$ \\
\hline
\end{tabular}

\section{DEVICE MODEL}

The role of material interface is for binding every layer to become a device. This operates for DC (Direct Current) parameters. Doped graphene channel is modeled with electrodes and six Carbon atom in a Si-C hexagonal structure. The carbon lattice layers are formed through covalently and establish strong carbon-carbon bonds [22]. The semiclassical Boltzmann transport model are used to confine channel width [26]. Contribution of electrons and holes for current conduction from source to drain happens simultaneously [6, 46]. The parameters used in the simulations are listed in Table 2 based on the extraction from different research papers. The 2D structure of GFET device modelled with mentioned parameters in Table 2 is shown in Figure 2 [38].

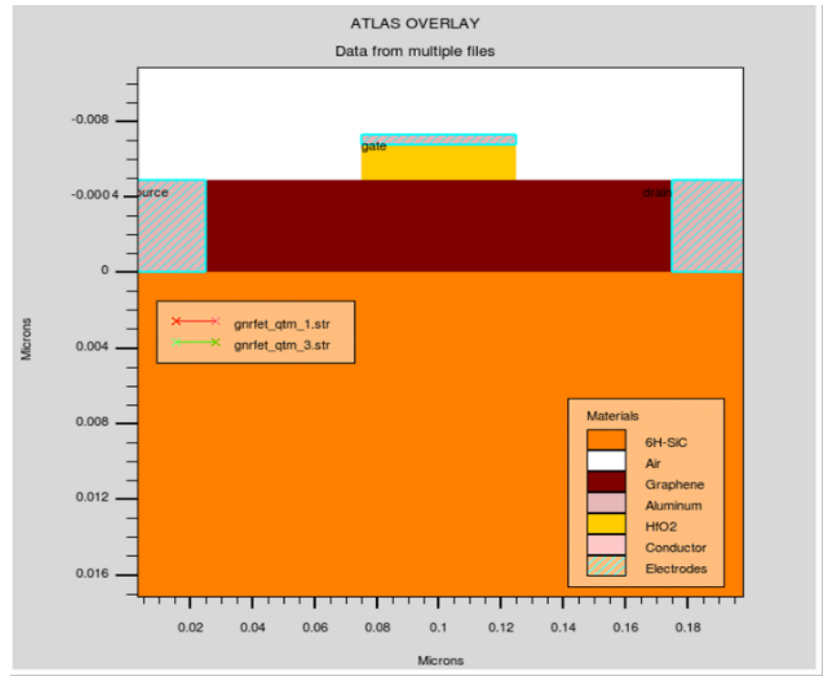

Figure 2: 2D-simulated GFET device.

Table 2: GFET device structure parameter

\begin{tabular}{|c|c|}
\hline Parameter & Data $(\mathbf{n m})$ \\
\hline Channel Thickness & 0.453 \\
\hline Channel Length & 150 \\
\hline Device Length & 200 \\
\hline Gate Dielectric Thickness & 6.4 \\
\hline
\end{tabular}

\section{RESULTS AND DISCUSSION}

\subsection{Analysis}

GFET operates under positive as well as negative gate voltage. This shows ambipolar behavior of GFET observed using Silvaco Atlas simulator [31, 48]. Also gate to substrate conductance, gate capacitance, parallel intrinsic capacitance, and parallel conductance were extracted through simulations etc. Dirac point at $1 \mathrm{~V} \mathrm{dc}$ gate bias voltage is shown in Figure 3 . The confinement of channel gate length purely depends on the gate capacitance. This is also called as quantum capacitance. The gate Quantum conductance is extracted between the substrate and the gate terminal. The measured substrate to gate parallel conductance is for applied gate voltage between $-0.2 \mathrm{~V}$ to $0.75 \mathrm{~V}$ respectively [3].

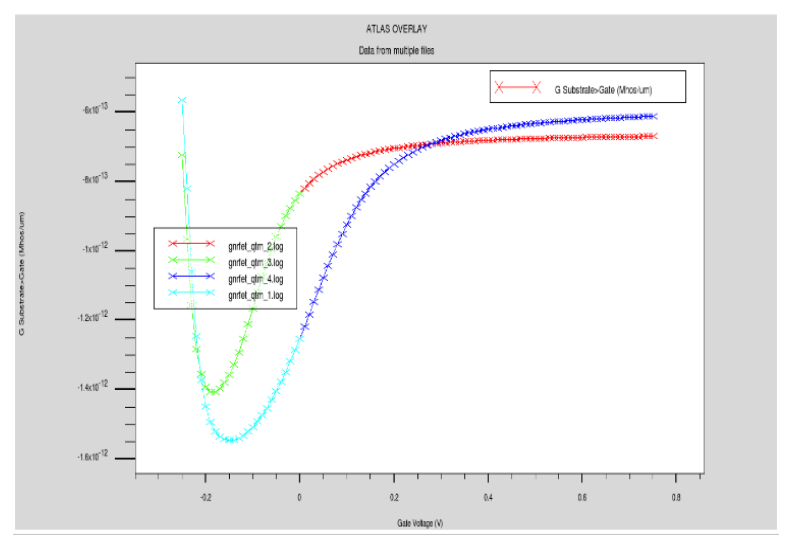

Figure 3: Gate conductance at confined channel region. 


\subsection{Gate Capacitance:}

At radio frequency transistor operates with three gate capacitances [41]. They are electrostatic capacitance, gate dielectric capacitance and quantum capacitance. This is extracted from the DC analysis and output transfer characteristics $[5,9]$. The smaller GFET with thinner gate oxide results in increased quantum mechanical effects [18, 37]. These are observed as shifts in threshold voltage and gate capacitance [28]. And were observed by varying gate voltage from -0.25 $\mathrm{V}_{\mathrm{DC}}$ to $+0.75 \mathrm{~V}_{\mathrm{DC}}$. Shown in figure 4 .

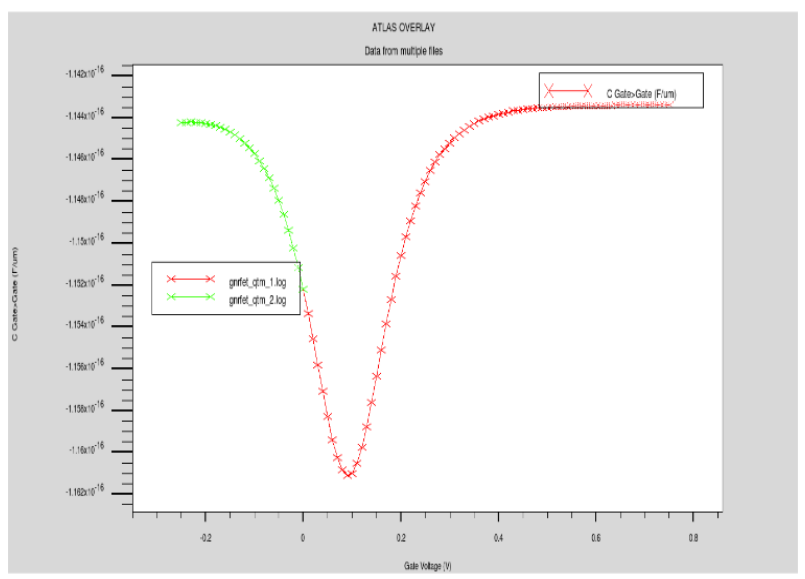

Figure 4: Gate dielectric capacitance

\subsection{Gate to source capacitance}

The gate to source capacitance is $4.61 \times 10^{\wedge}-17$ which is from grpah as shown in Figure 5. Here gate to source capacitance achieved desired to peek at $0.1 \mathrm{~V}_{\mathrm{DC}}$ with $-0.2 \mathrm{~V}_{\mathrm{DC}}$ to $+0.75 \mathrm{~V}_{\mathrm{DC}}$ voltage gate biased. This denotes the high-frequency incoming signal allowed by source terminal [47].

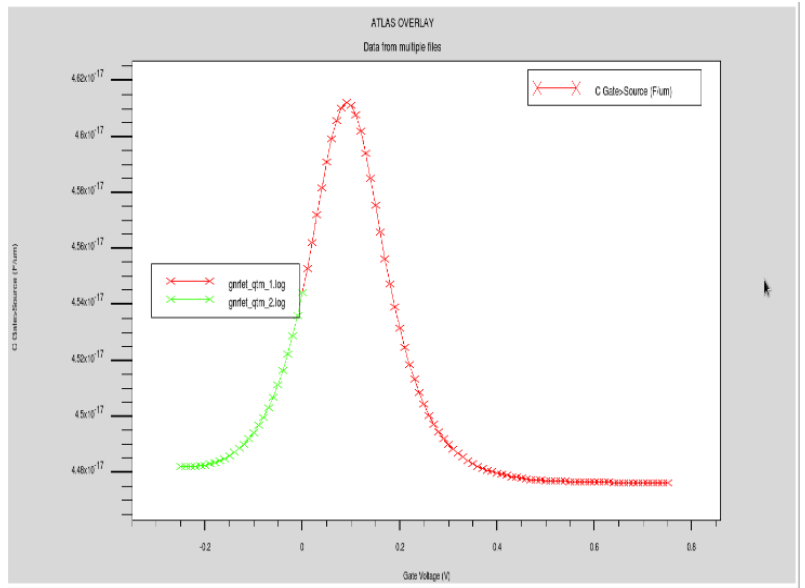

Figure 5: Parallel intrinsic capacitance

\subsection{Ambipolar behaviour of GFET}

Gate to substrate Capacitance extracted from the Figure 4 is more accurate at Dirac point for the multiplier circuit operation [3, 19, 31]. In the Figure 6 Dirac point measured while gate bias is from $-0.2 \mathrm{~V}_{\mathrm{DC}}$ to $+0.75 \mathrm{~V}_{\mathrm{DC}}$. This is exactly at the $+0.1 \mathrm{~V}$ gate voltage. Graphene parallel parallels to the device from the substrate to gate conductance measured as referrred in $[28,35]$.

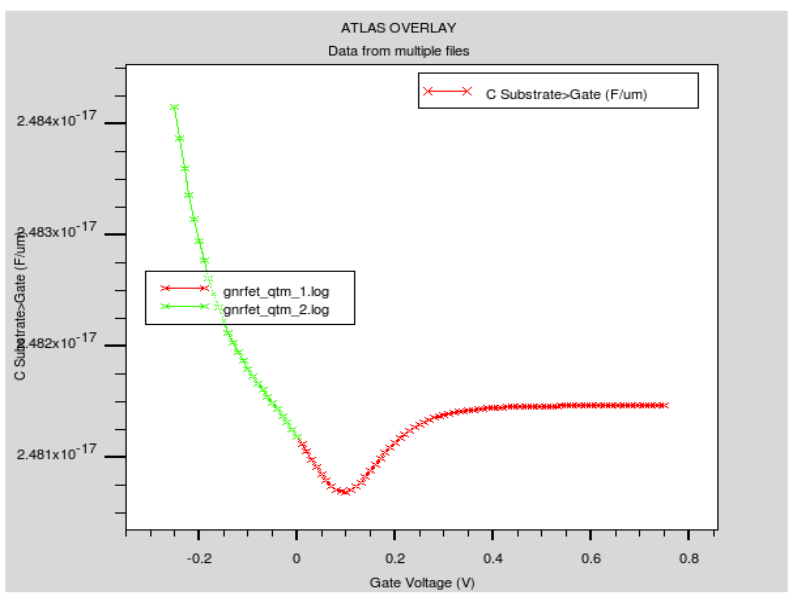

Figure 6: Parallel (substrate-Top gate) conductance

\subsection{Transfer curve}

The transfer characteristics for 50-nm gate shown in Figure 7. The single-channel GFET is simulated for a drain to source voltage $\left(V_{D S}\right)$ of $1 V_{D C}$. The transistor's threshold voltage $V_{\text {th }}$ is defined for $1 V_{D S}$ and a drain current of $10^{-7} \mathrm{~A}$ w/L flows and the effective gate-source voltage is related to the applied gatesource voltage $\mathrm{V}_{\mathrm{GS}}$. As to be expected from the $0.25 \mathrm{eV}$ bandgap of the channel. The transistors show excellent switchoff, and on-off ratio of $1.5 \times 10^{6}$ for a $1 \mathrm{~V}_{\mathrm{GS}}$ gate voltage swing (from $\mathrm{V}_{\mathrm{GS}}$ 'eff $=0.25 \mathrm{~V}_{\mathrm{DC}}$ to $+0.75 \mathrm{~V}_{\mathrm{DC}}$ ). The trans-conductance peaks at an effective gate voltage is around $0.68 \mathrm{~V}_{\mathrm{GS}}$.

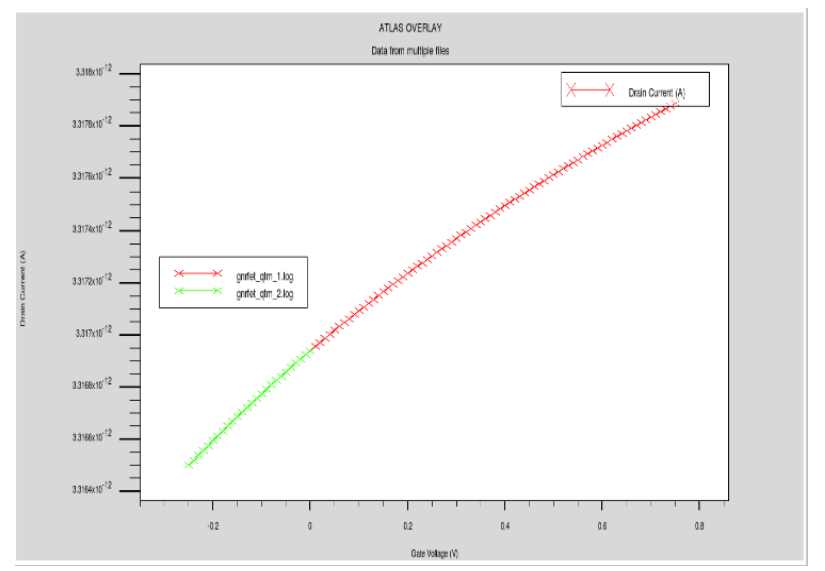

Figure 7: Transfer characteristics and

\subsection{Output Characteristics}

The output characteristics of transistor's operation and saturated drain current $I_{d}$ is shown in Figure 8. The low drain conductance is $1.66 \times \mathrm{e}^{-06}$ at a drain to source voltage $\left(\mathrm{V}_{\mathrm{DS}}\right)=$ $1 \mathrm{~V}_{\mathrm{DC}}$ and $\mathrm{V}_{\mathrm{GS}} \mathrm{seff}=0.5 \mathrm{~V}_{\mathrm{DC}}$ [46]. The good current saturation is caused by the semiconducting nature of the doped graphene channel. In addition to the high ON-OFF ratio, an important improvement in operation of GFETs in comparison to largearea graphene channels. The large are Graphene channels from a weak saturation and a large drain conductance $[10,30]$. 


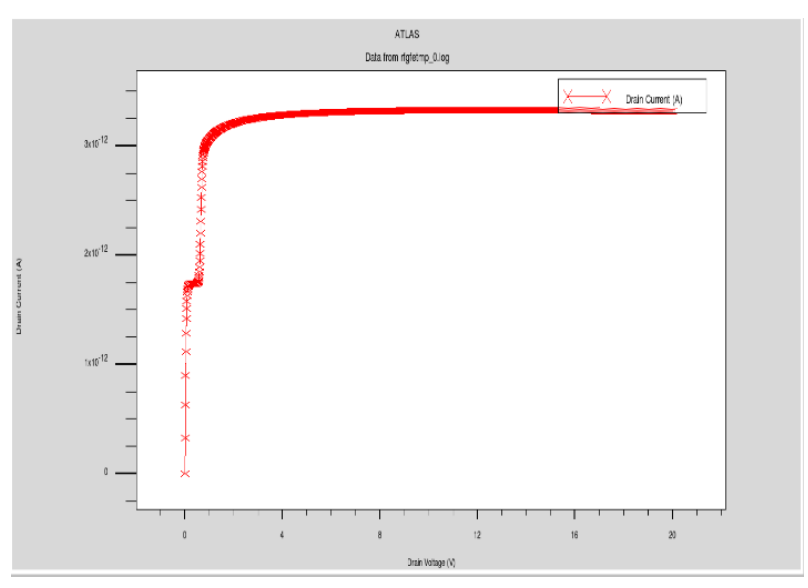

Figure 8: Output characteristics

\subsection{Intrinsic parameters}

As given in the Figure 10 radio frequency intrinsic parameters were extracted from the doped graphene transistor device simulator [43, 44]. These results are used to estimate the performance using the amplifier as a frequency multiplier circuit as shown in Figure 9 and 10 [29].

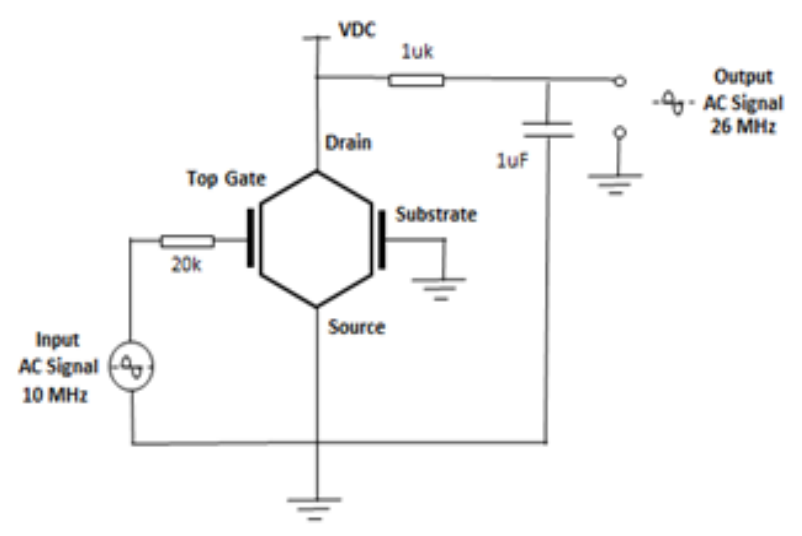

Figure 9: GFET frequency multiplier

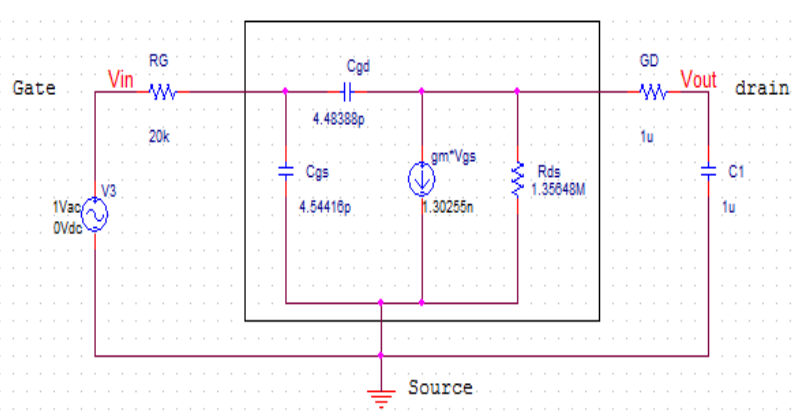

Figure 10: Equvalent circuit of GFET RF transistor

For the requirement of amplifier biasing, a voltage divider gate and drain resistors are include for separating all alternating current (AC) analysis data from the input and output circuit [9,
17, 29] as shown in Figure 11.The intrinsic parameter used in the simulation is given in Table 3 [7].

Table 3: Intrinsic GFET device parameters

\begin{tabular}{|c|c|}
\hline Intrinsic elements & DC parameters \\
\hline Gate to drain Capacitance & $4.48388 \mathrm{e}^{-17}$ \\
\hline Gate to source Capacitance & $4.54416 \mathrm{e}^{-17}$ \\
\hline Drain to source Resistance & $1.35648 \mathrm{e}^{+13}$ \\
\hline Trans-conductance & $1.30255 \mathrm{e}^{+13}$ \\
\hline
\end{tabular}

Band stop frequency depends on Gate to Drain Capacitance $\left(\mathrm{C}_{\mathrm{GD}}\right)$. This should be as small as possible, since the cut-off frequency will be high. Figure 7 shows the bode plot of the transistor's operation when it works as a low-pass filter.

This filter, only passes low frequency from. On the other hand, $[16,47]$. The frequency of the transistor amplifier always results in generation of HF signals. [23]. The cut-off frequency is between $10 \mathrm{MHz}$ to $30 \mathrm{MHz}$. High transmission bandwidth, Ultra low power and low complexity communication circuit are to be developed based on these results[24, 38].

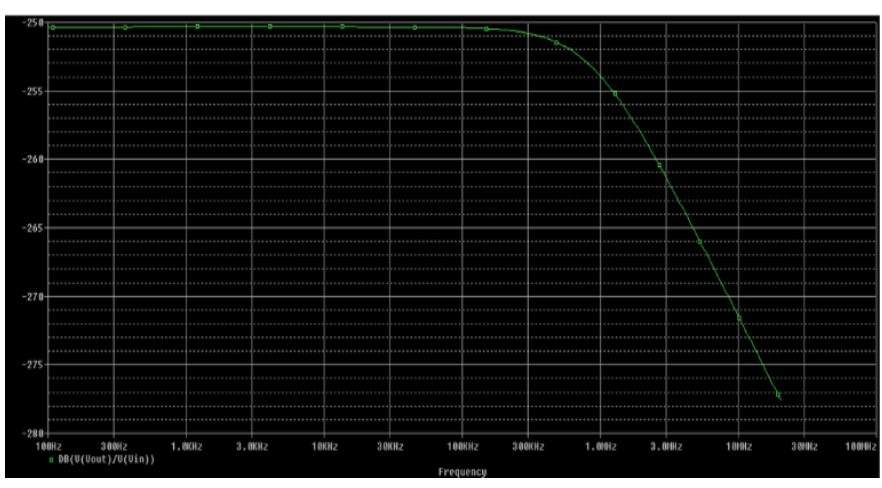

Figure 11: Bode plot shows highest cut-off frequency at maximum current gain $-275.5 \mathrm{~dB} /$ decade

\section{CONCLUSION}

The simulated results agree with standard observations found in the literature with regard to GFET with doped materials for channel. These simulations are to be tested with fabricated devices in the near future.

\section{REFERENCES}

[1] Nanmeni Bondja, C., Geng, Z., Granzner, R., Pezoldt, J., \& Schwierz, F. (2016). Simulation of 50-nm gate graphene nanoribbon transistors. Electronics, 5(1), 3.

[2] Al-Amin, C., Karabiyik, M., Vabbina, P. K., Sinha, R., \& Pala, N. (2016). Graphene FETs with LowResistance Hybrid Contacts for Improved High Frequency Performance. Nanomaterials, 6(5), 86. 
[3] Cheng, C., Huang, B., Liu, J., Zhang, Z., Mao, X., Xue, P., \& Chen, H. (2016). A pure frequency tripler based on CVD graphene. IEEE Electron Device Letters, 37(6), 785-788.

[4] Lu, N., Wang, L., Li, L., \& Liu, M. (2017). A review for compact model of graphene field-effect transistors. Chinese Physics B, 26(3), 036804.

[5] Granzner, R., Thiele, S., Schippel, C., \& Schwierz, F. (2010). Quantum effects on the gate capacitance of trigate SOI MOSFETs. IEEE Transactions on Electron Devices, 57(12), 3231-3238.

[6] Pasadas, F., \& Jiménez, D. (2016). Large-signal model of graphene field-effect transistors-Part I: Compact modeling of GFET intrinsic capacitances. IEEE Trans. Electron Devices, 63(7), 2936-2941.

[7] Pasadas, F., \& Jiménez, D. (2016). Large-signal model of graphene field-effect transistors-Part II: Circuit performance benchmarking. IEEE Transactions on Electron Devices, 63(7), 2942-2947.

[8] Gunlycke, D., \& White, C. T. (2008). Tight-binding energy dispersions of armchair-edge graphene nanostrips. Physical Review B, 77(11), 115116.

[9] Andersson, M. A., Habibpour, O., Vukusic, J., \& Stake, J. (2012). Resistive graphene FET subharmonic mixers: Noise and linearity assessment. IEEE Trans. Microw. Theory Techn, 60(12), 4035-4042.

[10] Attiya, A. M. (2016, February). Modeling nonlinear electrical conductivity of graphene by using finite difference time domain. In Radio Science Conference (NRSC), 2016 33rd National (pp. 340-347). IEEE.

[11] Attiya, A. M. (2012, April). B1. Nanotechnology in $\mathrm{RF}$ and microwave applications. In Radio Science Conference (NRSC), 2012 29th National (pp. 9-18). IEEE.

[12] Awan, S. A., Lombardo, A., Colli, A., Privitera, G., Kulmala, T. S., Kivioja, J. M. \& Ferrari, A. C. (2016). Transport conductivity of graphene at RF and microwave frequencies. 2D Materials, 3(1), 015010.

[13] Beshkova, M., Hultman, L., \& Yakimova, R. (2016). Device applications of epitaxial graphene on silicon carbide. Vacuum, 128, 186-197.

[14] Brookner, E. (2016, May). Developments and breakthroughs in radars and phased-arrays. In Radar Conference (RadarConf), 2016 IEEE (pp. 1-6). IEEE.

[15] Colombo, L., Wallace, R. M., \& Ruoff, R. S. (2013). Graphene growth and device integration. Proceedings of the IEEE, 101(7), 1536-1556.

[16] Al-Amin, C., Karabiyik, M., Vabbina, P. K., Sinha, R., \& Pala, N. (2014). Field controlled RF Graphene FETs with improved high frequency performance. Solid-State Electronics, 95, 36-41.
[17] Ellinger, F., Claus, M., Schröter, M., \& Carta, C. (2011, October). Review of advanced and beyond CMOS FET technologies for radio frequency circuit design. In Microwave \& Optoelectronics Conference (IMOC), 2011 SBMO/IEEE MTT-S International (pp. 347-351). IEEE.

[18] Ezhilvalavan, S., Tsai, M. S., \& Tseng, T. Y. (2000). Dielectric relaxation and defect analysis of Ta2O5 thin films. Journal of Physics D: Applied Physics, 33(10), 1137.

[19] Fu, W., Feng, L., Mayer, D., Panaitov, G., Kireev, D., Offenha $\square$ usser, A., \& Krause, H. J. (2016). Electrolyte-gated graphene ambipolar frequency multipliers for biochemical sensing. Nano letters, 16(4), 2295-2300.

[20] Grande, M., Bianco, G. V., Vincenti, M. A., de Ceglia, D., Capezzuto, P., Petruzzelli, V., \& D'Orazio, A. (2016, July). Graphene-based devices: A platform for high frequency applications?. In Transparent Optical Networks (ICTON), 2016 18th International Conference on (pp. 1-4). IEEE.

[21] I. N. Hajj, (2015), On Device Modeling for Circuit Simulation With Application to Carbon-Nanotube and Graphene Nano-Ribbon Field-Eff ect Transistors, vol. 34, no. 3, pp. 495499.

[22] Ishida, K., Meister, T., Shabanpour, R., Boroujeni, B. K., Carta, C., Cantarella, G., \& Ellinger, F. (2016, July). Radio frequency electronics in a-IGZO TFT technology. In Active-Matrix Flatpanel Displays and Devices (AMFPD), 2016 The 23rd International Workshop on Active-Matrix Flatpanel Displays and Devices (pp. 273-276). IEEE.

[23] S. Joshi, E. Shitaljoshimyuntedu, and E. Kougianos, (2016) Unconventional EDA for Mixed-Signal Circuits: A Graphene FET based LC-VCO Case Study, no. 1, pp. 23.M. June, No Title, pp. 7679.

[24] M. Karabiyik and N. Pala, (2016) Microelectronic Engineering Fabrication of Graphene Field-effect Transistor with Field Controlling Electrodes to improve f T, MEE, vol. 164, pp. 7174.

[25] T. J. Kazmierski, I. J. Umoh, S. Member, T. J. Kazmierski, and S. Member, (2016) 'A dual-gate graphene FET model for circuit simulation - SPICE implementation A Dual-Gate Graphene FET Model for Circuit Simulation SPICE Implementation, no. October.

[26] Y. Korniyenko and O. Shevtsov, (2016). Nonlinear response of a ballistic graphene transistor with an acdriven gate: High harmonic generation and terahertz detection, vol. 125445, pp. 115.

[27] J. Lee, T. Ha, K. N. Parrish, S. Member, and S. F. Chowdhury, (2013) High-Performance Current Saturating Graphene Field-Eff ect Transistor With Hexagonal Boron Nitride Dielectric on Flexible Polymeric Substrates, vol. 34, no. 2, pp. 172174. 
[28] C. Link, H. Wang, A. Hsu, D. S. Lee, K. K. Kim, J. Kong, and T. Palacios, (2016) Delay Analysis of Graphene Field-Eff ect Transistors, pp. 36.

[29] H. Madan, S. Member, V. Saripalli, H. Liu, S. Datta, and S. Member, (2012). Asymmetric Tunnel FieldEff ect Transistors as Frequency Multipliers, vol. 33, no.11, pp. 15471549.

[30] I. Maeng, S. Lim, S. J. Chae, Y. H. Lee, H. Choi, and J. Son, (2012) Gate-Controlled Nonlinear Conductivity of Dirac Fermion in Graphene FieldEff ect Transistors Measured by Terahertz TimeDomain Spectroscopy.

[31] J. S. Moon, D. Curtis, D. Zehnder, S. Kim, D. K. Gaskill, G. G. Jernigan, C. R. Eddy, P. M. Campbell, K. Lee, and P. Asbeck, (2011) Low-Phase-Noise Graphene FETs in Ambipolar RF Applications, vol. 32 , no. 3, pp. 270272.

[32] J. S. Moon, M. Antcliff e, H. C. Seo, S. C. Lin, A. Schmitz, I. Milosavljevic, K. McCalla, D. Wong, D. K. Gaskill, P. M. Campbell, K. M. Lee, and P. Asbeck, (2012). Graphene review: An emerging RF technology, 2012 IEEE 12th Top. Meet. Silicon Monolith. Integr. Circuits RF Syst. SiRF 2012 - Dig. Pap., pp. 199202.

[33] J. S. Moon, D. Curtis, M. Hu, D. Wong, C. McGuire, P. M. Campbell, G. Jernigan, J. L. Tedesco, B. VanMil, R. Myers-Ward, C. Eddy, and D. K. Gaskill,(2009). Epitaxial-graphene RF field-eff ect transistors on Si-face $6 \mathrm{H}-\mathrm{SiC}$ substrates, IEEE Electron Device Lett., vol. 30, no. 6, pp. 650652.

[34] J. Moon and D. K. Gaskill, (2011). Graphene: Its Fundamentals to Future Applications, vol. 59, no. 10, pp. 27022708.

[35] J. Moon, D. K. Gaskiu, P. Campben, and P. Asbeck, 90265 2), no. c, pp. 36. 32 Y. Nagahisa, Y. Harada, and E. Tokumitsu, (2013) Unipolar behavior in graphene channel field-eff ect-transistors with n-type doped SiC source / drain regions, vol. 223503.

[36] D. Park, T. Chang, S. Mikael, J. Seo, and P. F. Nealey, (2013) Graphene RF transistors with Buried Bottom Gate, pp. 8486.

[37] S. Park, S. H. Shin, M. N. Yogeesh, A. L. Lee, and S. Rahimi, (2016) Extremely High Frequency Flexible Graphene Thin Film Transistors, no. c, pp. 14.

[38] S. Park, W. Zhu, H. Chang, M. N. Yogeesh, R. Ghosh, S. K. Banerjee, and D. Akinwande, (2015), 'HighFrequency Prospects of 2D Nanomaterials for Flexible Nanoelectronics from Baseband to $\mathrm{Sub}-\mathrm{THz}$ Devices', pp. 827830.

[39] L. Pierantoni and F. Coccetti, (2010) Radio-frequency nanoelectronics: A new paradigm in electronic systems design, Asia-Pacific Microw. Conf., pp. 10071014.
[40] Prajapati, D. D., \& Neelkanthan, U. (2017). Review Paper on Frequency Multiplier at Terahertz Range. International Journal on Recent and Innovation Trends in Computing and Communication, 5(2), 228232.

[41] Russer, P., \& Fichtner, N. (2010). Nanoelectronics in radio-frequency technology. IEEE Microwave Magazine, 11(3), 119-135.

[42] Schroter, M., Claus, M., Sakalas, P., Wang, D., \& Haferlach, M. (2012, September). An overview on the state-of-the-art of carbon-based radio-frequency electronics. In Bipolar/BiCMOS Circuits and Technology Meeting (BCTM), 2012 IEEE (pp. 1-8). IEEE.

[43] Schwierz, F. (2010). Graphene transistors. Nature nanotechnology, 5(7), 487.

[44] Schwierz, F. (2010, November). Graphene transistors-A new contender for future electronics. In Solid-State and Integrated Circuit Technology (ICSICT), 2010 10th IEEE International Conference on (pp. 1202-1205). IEEE.

[45] Shkerdin, G., Alkorre, H., Guoqiang, H., \& Stiens, J. (2016). Modified TE modes of metal waveguide with integrated graphene structure in the sub-terahertz frequency range. IET Microwaves, Antennas \& Propagation, 10(6), 692-699..

[46] Song, S. M., Bong, J. H., Hwang, W. S., \& Cho, B. J. (2016). Improved drain current saturation and voltage gain in graphene-on-silicon field effect transistors. Scientific reports, 6, 25392.

[47] Hooge, F. N., Kleinpenning, T. G. M., \& Vandamme, L. K. J. (1981). Experimental studies on 1/f noise. Reports on progress in Physics, 44(5), 479.

[48] Silvaco Int. (2006) ATLAS User's Manual, Device Simulation Software, www.silvaco.com 